
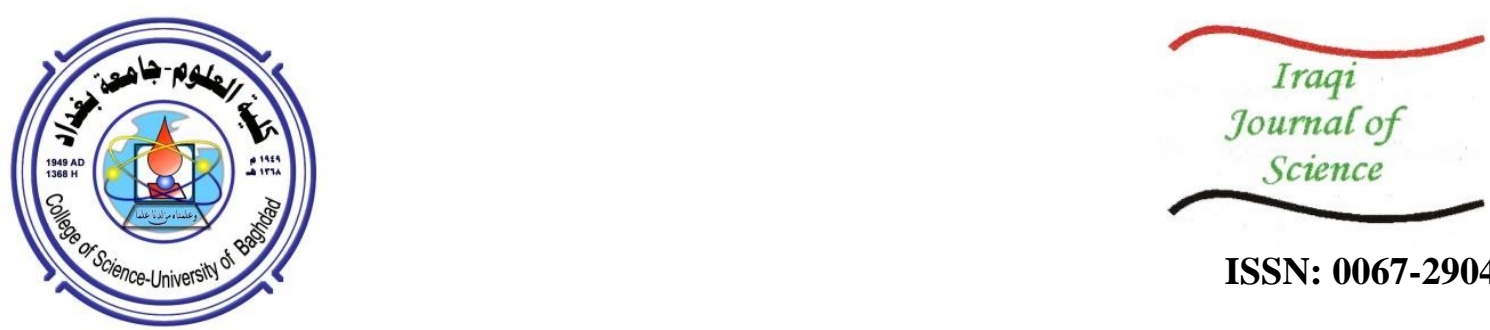

ISSN: 0067-2904

\title{
The Effect of Polarization Flipping Point on Polarization Dynamics by Optical Feedback Technique
}

\author{
Ehsan Ali Abed ${ }^{1}$, Jassim Mohammed Najim², Suaded Salman Ahmed ${ }^{2}$ \\ ${ }^{1}$ Department of Physics, University of Anbar, Anbar, Iraq \\ ${ }^{2}$ Department of Physics, University of Baghdad, Baghdad, Iraq \\ Received: 10/12/2019 \\ Accepted: 29/5/2020

\begin{abstract}
The effect of the optical feedback on the polarization flipping point and hysteresis loop was studied. The polarization flipping occurred at all angles between the polarizer axis and the laser polarization. The polarization flipping point changed by an optical feedback occurred at angles from $0^{\circ}$ to $90^{\circ}$. Ability of choosing or controlling the laser polarization was determined by changing the direction of vertical and horizontal polarization by polarizer rotation in the external cavity from $0^{\circ}$ to $90^{\circ}$.
\end{abstract}

Keywords: Laser; Optical feedback; Polarization flipping point; hysteresis loop

$$
\begin{aligned}
& \text { تأثير نقطة تقلب الاستقطاب على ديناميكية الاستقطاب بواسطة تقنية التغذية العكسية البصرية } \\
& \text { احسان علي عبيد، جاسم عحم نجم²، سوؤدد سلمان احمد } 2 \\
& \text { 1قسم الفيزياء، جامعة الانبار ، الانبار ، العراق }
\end{aligned}
$$

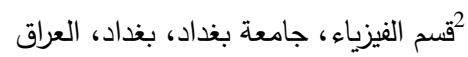$$
\text { الخلاصة }
$$

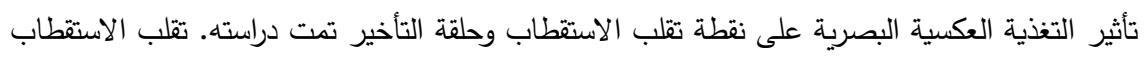

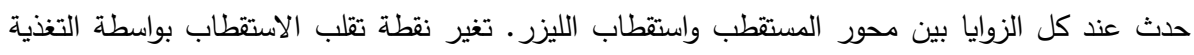

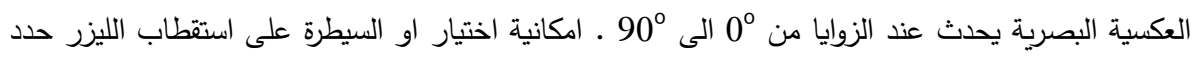

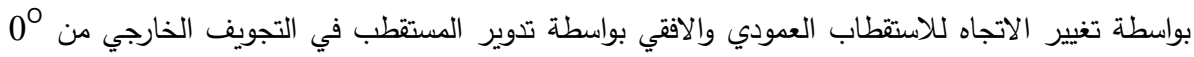$$
\text { الى } 90^{\circ}
$$

\section{Introduction}

Polarization is a very fundamental property of light that is necessary to consider for the vertical characteristics of electromagnetic waves. Many approaches in optics are decided through the polarization states of the considered beams. Recently, studies on the polarization of light in lasers with optical feedback have attracted remarkable attention [1-4]. Floch et al. observed polarization switches and a hysteresis effect via changing the intracavity anisotropy values of the laser [1,5]. Stephan et al. experimentally and theoretically studied the polarization adjustments brought about by means of optical feedback from a polarizer that is external to the cavity [6].

With the aid of the reflecting part of the laser output back into the resonant cavity, the conduct of the laser, in particular the static and dynamic residences, can be appreciably affected. This is called the optical feedback effect, or self-mixing interference, which was first suggested by king and Steward 
[7]. Theoretically, and to verify experimentally the polarization switching techniques happening in a gas laser when an internal parameter along with the laser frequency as an example turned into various [8].

This paper includes the following steps:

1. The polarization flipping point leads to the production of the hysteresis loop (HL) between the horizontal $(/ /)$ and vertical $(\perp)$ polarization for the He-Ne laser. The HL leads to a decrease in the two components of intensity $\left(\mathrm{I}_{/ /}\right.$and $\left.\mathrm{I}_{\perp}\right)$.

2. This problem is treated by optical feedback from the external cavity or reflector $\left(\mathrm{M}_{3}\right)$.

3. In previous investigations, researchers took few angles for optical feedback by polarizer rotation in the external cavity for the treatment of this problem.

4. In our paper, we took multiple angles for optical feedback by polarizer rotation in the external cavity. Therefore, the two components intensity values $\left(I_{/ /}\right.$and $I_{\perp}$ ) became high and the HL size became small. The results in our research are remarkably different from those in previous studies.

\section{Experimental setup}

Figure-1 shows the layout of experimental setup. The setup consists of three elements, namely the feedback, laser, and detection elements.

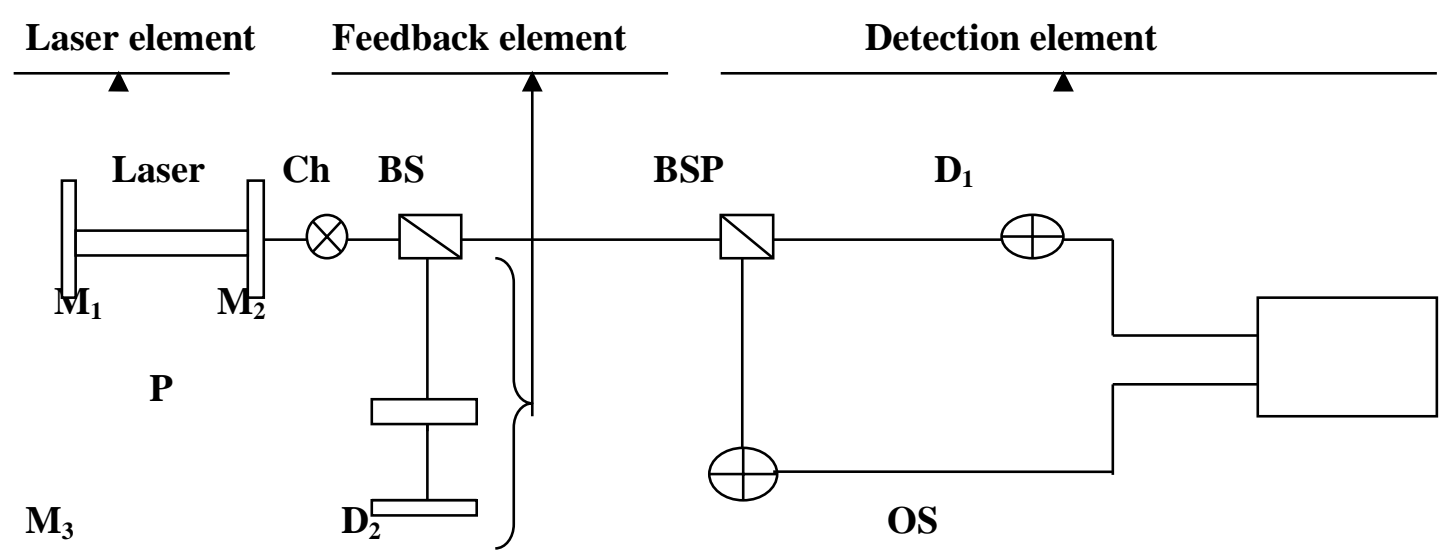

Figure 1-The Layout of experimental setup and unique coordinates. $\mathrm{M}_{1}, \mathrm{M}_{2}$ cavity mirrors; $\mathrm{Ch}$, chopper; BS, beam splitter; $\mathrm{P}$, rotating polarizer; $\mathrm{M}_{3}$, feedback mirror; PBS, polarizing beam splitter ; $\mathrm{D}_{1}, \mathrm{D}_{2}$, photo detectors; OS, digital storage oscilloscope.

The intra-cavity He-Ne laser generates a linear polarization with a single longitudinal mode oscillating in the resonant cavity. The working wavelength is $632.8 \mathrm{~nm}$. The gaseous pressure ratios in the laser tube are $\mathrm{He}: \mathrm{Ne}=9: 1$ and $\mathrm{Ne} 20: \mathrm{Ne} 22=1: 1$. The reflectivity values of the cavity mirrors $\mathrm{M}_{1}$ and $\mathrm{M}_{2}$ are $99.99 \%$ and $99 \%$, respectively. The length of the resonant cavity formed by the mirrors M1 and $\mathrm{M} 2$ is $40 \mathrm{~cm}$ [9].

The feedback element is made of a chopper Ch., a feedback mirror $\mathrm{M}_{3}$, and a polarizer P. The feedback cavity length is about $60 \mathrm{~cm}$. The reflectivity of $\mathrm{M}_{3}$ is $99.99 \%$. The angle between the $\mathrm{X}$ axis and the optical axis of the polarizer is denoted by $\theta$. The light whose polarization is parallel to the $\mathrm{X}$ axis is denoted by //-polarization, and the other one is denoted by $\perp$-polarization.

The detection element consists of a polarizing beam splitter PBS, photo detectors $\mathrm{D}_{1}, \mathrm{D}_{2}$, and a digital storage oscilloscope OS. The $\perp$-polarization and //-polarization are separated by the PBS, and their intensities $\left(\mathrm{I}_{\perp}\right.$ and $\left.\mathrm{I}_{/ /}\right)$are detected by $\mathrm{D}_{1}, \mathrm{D}_{2}$, and OS, respectively.

\section{Theoretical analysis}

A theoretical version based totally on self-consistency of the laser became available. Helium-neon (He-Ne) laser oscillates in two orthogonally polarized modes in the resonant cavity. The two modes transmit through $\mathrm{M}_{2}$ and are reflected by the beam splitter to the polarizer. Firstly, the //-mode transmits through the polarizer to the feedback mirror $\mathrm{M}_{3}$ and then it reflects by $\mathrm{M}_{3}$ into the resonant cavity again. From the intra cavity, the electric vector $E_{/ / 1}(t)$, and from the external cavity, the electric vector $E_{/ / 2}(t)$, combine together into the intra cavity to obtain $E_{/ /}(t)$ and, hence, the feedback effect is generated [9]. 


$$
\begin{array}{r}
E_{/ /}(t)=E_{/ / 1}(t)+E_{/ / 2}(t) \ldots \\
E_{/ / 1(t)}=r_{1} r_{2} \exp \left(i 4 \pi v_{/ /} L / c+2 g_{/ /} L\right) E_{/ / o}(t) \ldots \ldots \\
E_{/ / 2(t)}=T_{c h} r_{b s} T_{p} r_{2} r_{3} T_{2}^{2} M_{/ /} \exp \left[i 4 \pi v_{/ /}(L+l) / c+2 g_{/ /} L\right] E_{/ / o}(t)
\end{array}
$$

According to the self-consistency of the laser, the following equation is produced

$$
\begin{array}{r}
E_{/ /}(t)=E_{/ / o}(t) \ldots \ldots \ldots \ldots \ldots(4) \\
\mathrm{E}_{/ /}(\mathrm{t})=\mathrm{r}_{1} \mathrm{r}_{2} \exp \left(\mathrm{i} 4 \pi v / / \mathrm{L} / \mathrm{c}+2 g_{/ /} \mathrm{L}\right) \mathrm{E}_{/ / \mathrm{o}}(\mathrm{t})+\mathrm{T}_{\mathrm{ch}} \mathrm{r}_{\mathrm{bs}} \mathrm{T}_{\mathrm{p}} \mathrm{r}_{1} \mathrm{r}_{3} \mathrm{t}_{2}^{2} M_{/ /} \exp \left[\mathrm{i} 4 \pi v_{/ /}(\mathrm{L}+\mathrm{l}) / \mathrm{c}+\right. \\
\left.2 g_{/ /} \mathrm{L}\right] \mathrm{E}_{/ / \mathrm{o}}(\mathrm{t}) \ldots \ldots \ldots(5)
\end{array}
$$

where $r_{1}, r_{2}, r_{3}$ and $\mathrm{r}_{\mathrm{bs}}$ are the reflection coefficient of $\mathrm{M}_{1}, \mathrm{M}_{2}, \mathrm{M}_{3}$, and the beam splitter, respectively, $t_{2}$ is the transmission coefficient of $\mathrm{M}_{2}$, and $T_{\mathrm{p}}$ and $\mathrm{T}_{\mathrm{ch}}$ are the transmissivity values of the polarizer and chopper, respectively. $M_{/ /}$is the factor brought about through Jones matrix for polarizers [7]

$$
\mathrm{M}_{/ /}=\cos ^{4} \theta+\sin ^{2} 2 \theta / 4
$$

$v_{\|}$is the frequency of horizontal polarization (//-polarization). $L$ and $l$ are the laser cavity and the feedback cavity lengths, respectively. $g$ is the linear gain per unit length due to the simulated emission inside the resonant cavity with the availability of the external feedback.

Equations (4) and (5) can be solved to obtain [10-12]

$$
\begin{gathered}
\Delta g=-\beta M \cos \left(4 \pi \frac{v l}{c}\right) \\
\Delta g_{/ /}=-\Delta g_{\perp}=-\beta M \cos \left(4 \pi \frac{v l}{c}\right) \\
\Delta g_{/ /}=-\beta M_{/ /} \cos \left(4 \pi v_{/ /} l / c\right)
\end{gathered}
$$

where $\beta=\mathrm{T}_{\text {ch }} \mathrm{r}_{\mathrm{bs}} \mathrm{T}_{\mathrm{p}} \mathrm{r}_{3} \mathrm{t}_{2}^{2} / \mathrm{r}_{2}$$$
\Delta g_{/ /}=g_{/ /}-g_{/ / o}
$$

where $g_{/ /}$and $g_{/ / o}$ represent the gain with and without feedback, respectively. When vertical polarization ( $\perp$-polarization) oscillates, the following equation can be obtained

$$
\Delta g_{\perp}=\beta M_{\perp} \cos \left(4 \pi v_{\perp} l / c\right)
$$

$\Delta g_{\perp}=g_{\perp}-g_{\perp o}$

where $g_{\perp}$ and $g_{\perp o}$ represent the gain with and without feedback, respectively.

$M_{\perp}$ is the factor brought about through Jones matrix for polarizers

$$
M_{\perp}=\sin ^{4} \theta+\sin ^{2} 2 \theta / 4
$$

As illustrated in Eqs. (9) and (10), $\Delta g_{/ /}$and $\Delta g_{\perp}$ are both modulated by $\theta$ (from the rotating polarizer) and feedback cavity length $l$. Furthermore, there is a phase difference between $\Delta g_{/ /}$and $\Delta g_{\perp}$ due to the frequency difference between $v_{/ /}$and $v_{\perp}$. By supposing that $v_{/ /}$is larger than $v_{\perp}$, $\Delta g_{/ /}-\Delta g_{\perp}$ is denoted as $G_{\Delta}$ which may be expressed as in eq . (12)

$$
G_{\Delta}=\beta\left[M_{/ /} \cos \left(\alpha+\frac{2 \pi \Delta v l}{c}\right)-M_{\perp} \cos \alpha\right]
$$

where $\quad \alpha=4 \pi v_{\perp} l / c . G_{\Delta}$ is modulated by $l, \theta$ and $\Delta v$. The amplitude of $G_{\Delta}$ is denoted as $G$, which is modulated by $\theta$ and $\Delta v$. 

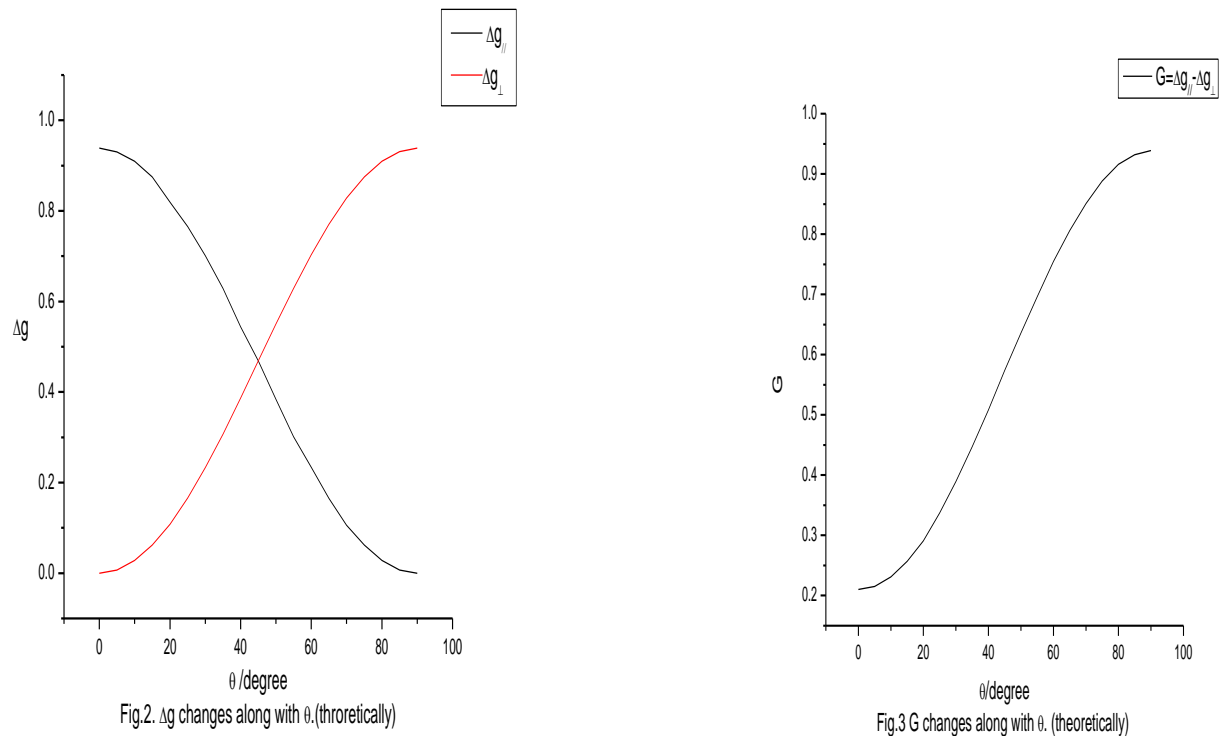

Figure 2-indicates that $\Delta g$ changes with $\theta$ from the rotating polarizer. $\Delta g / /$ for horizontal polarization increases at $\theta=0$ then decreases at $\theta=90 . \Delta g_{\perp}$ for vertical polarization decreases at $\theta=0$ then increases at $\theta=90$. The polarization flipping occurs at $\theta=45$. Figure- 3 indicates that $G$ changes along with $\theta$. Also, $\mathrm{G}$ increases with the increase of $\theta$. There is no polarization flipping at any angle.

The laser intensity with optical feedback can be expressed as [13]

$$
I=I_{o}(1-k \Delta g)
$$

where $\mathrm{k}$ is a constant relating to the laser operation parameters and $I_{o}$ is the initial laser intensity without optical feedback.

By substituting Eq. (9) and Eq.(10) into Eq.(13), the intensities of the two modes with optical feedback can be written as

$$
\begin{aligned}
& I_{/ /}=I_{/ / o}\left[1+k_{/ /} \beta M_{/ /} \cos \left(\frac{4 \pi v_{/ /} l}{c}\right)\right] \\
& I_{\perp}=I_{\perp \mathrm{o}}\left[1-k_{\perp} \beta M_{\perp} \cos \left(\frac{4 \pi v_{\perp} l}{c}\right)\right]
\end{aligned}
$$

where $I_{/ / o}$ and $I_{\perp \circ}$ are the intensities of the two polarized modes without optical feedback.

$I_{/ /}$and $I_{\perp}$ are the intensities of the two polarized modes with optical feedback.
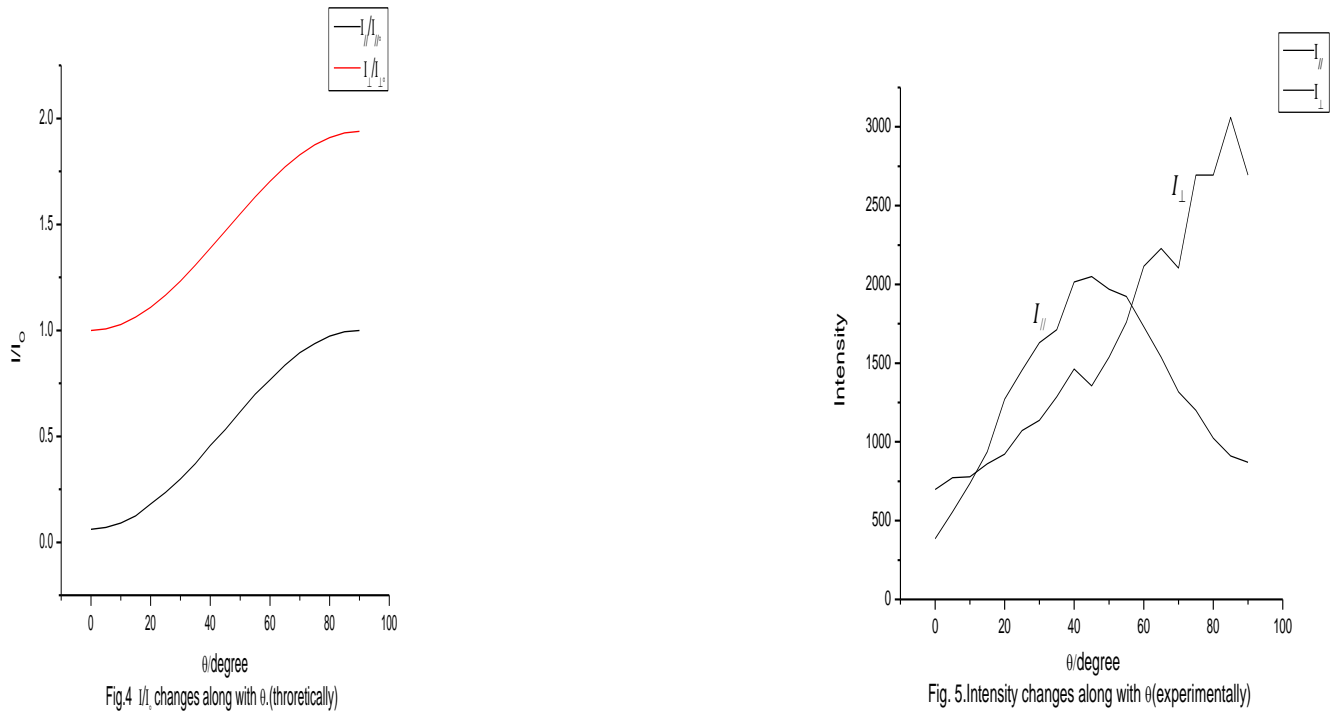

Figure 4-shows that $\frac{I_{\perp}}{I_{\perp o}} \geq \frac{I_{/ /}}{I_{/ / o}}$ with the optical feedback. This theoretical result is identical to the practical result in Figure- 5, where $I_{\perp}$ with the optical feedback is much greater than the $I_{/ /}$with the optical feedback at $90^{\circ}$. Also, there is no polarization flipping between $\mathrm{I}_{/ /} / \mathrm{I}_{/ / o}$ and $\mathrm{I}_{\perp} / \mathrm{I}_{\perp \mathrm{o}}$ (theoretically). 
Figure-5 shows that polarization flipping occurs between $\mathrm{I}_{/ /}$and $\mathrm{I}_{\perp}$ at $\theta=10^{\circ}$ and $\theta=60^{\circ}$ with optical feedback. Also, $\mathrm{I}_{\perp} \geq \mathrm{I}_{/ /}$at $\theta=90^{\circ}$ with optical feedback (experimentally ).

\section{Results and discussion}

Figures- 6 to 14 show that the polarization flipping from x-to-y is obtained without and with optical feedback. Figure-6 shows horizontal(//) and vertical $(\perp)$ polarization without optical feedback. One can observe that $I_{/ /}$and $I_{\perp}$ intensity are stable and that the polarization flipping point does not move because of the absence of the optical feedback. Figure-7 shows that //- polarization oscillates and $\mathrm{I}_{/ /}$is modulated by optical feedback because of the combination between $\mathbf{E}_{/ / 1}(\mathbf{t})$ and $\mathbf{E}_{/ / 2}(\mathbf{t})$ into the intra cavity. While $\perp$-polarization does not oscillate, $\mathrm{I}_{\perp}$ is stable because of the presence of the polarizer. In this case, the polarization flipping point moves to the right. When the polarization flipping point moves to the right, $\mathrm{I}_{/ /}$increases and the hysteresis loop decreases.

In Figures- 8 to 11 , it is shown that $\mathrm{I}_{/ /}$increases and the hysteresis loop decreases, while $\mathrm{I}_{/ /}$reaches to saturation state. Figures- 12, 13, and 14 show that $\perp$-polarization oscillates and $\mathrm{I}_{\perp}$ increases because of the combination between $\mathbf{E}_{\perp 1}(\mathbf{t})$ and $\mathbf{E}_{\perp 2}(\mathbf{t})$ into the intra cavity. Figures- 15 to 25 show that the polarization flipping from $\mathrm{y}$-to-x is obtained. Figure-15 shows that the polarization flipping point moves to the left, $I_{\perp}$ increases, and the hysteresis loop decreases. Figures-16 to 25 show that $I_{\perp}$ increases and the hysteresis loop decreases, while $\mathrm{I}_{\perp}$ reaches to the saturation state.

$\mathrm{I}_{\perp}$ is higher than $\mathrm{I}_{/ / /}$and the hysteresis loop does not disappear at //- polarization oscillation, while it disappears at $\perp$-polarization oscillation.
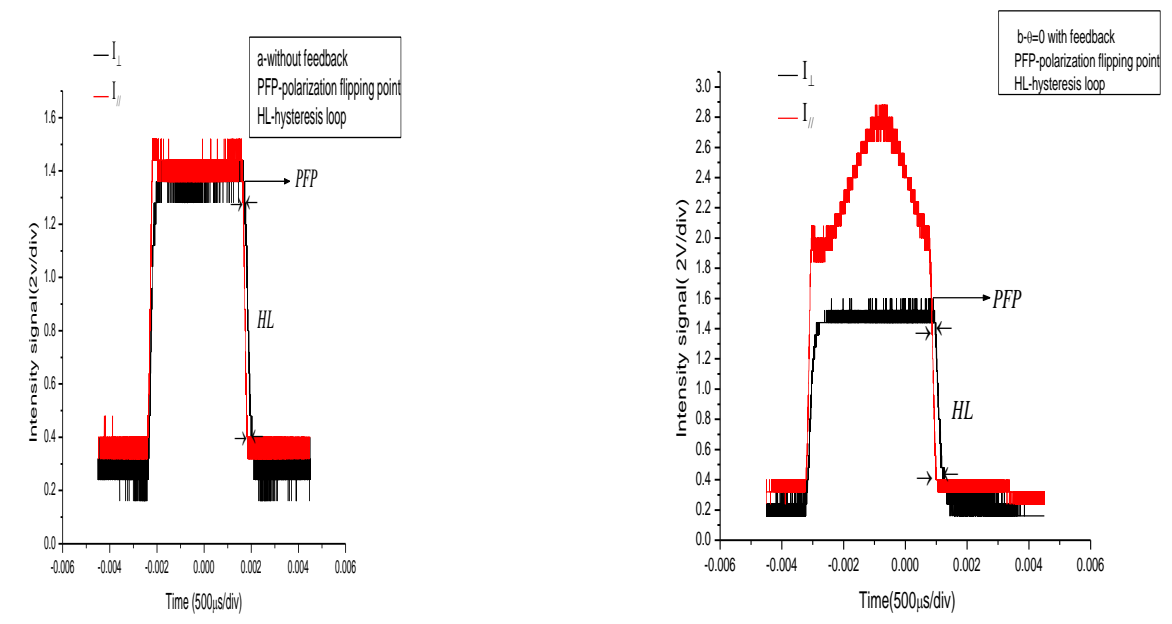

Figure 6-The intensities of $I_{/ /}$and $I_{\perp}$ without optical Figure 7- The intensities of $I_{/ /}$and $I_{\perp}$ with optical feedback feedback at $\theta=0^{\circ}$

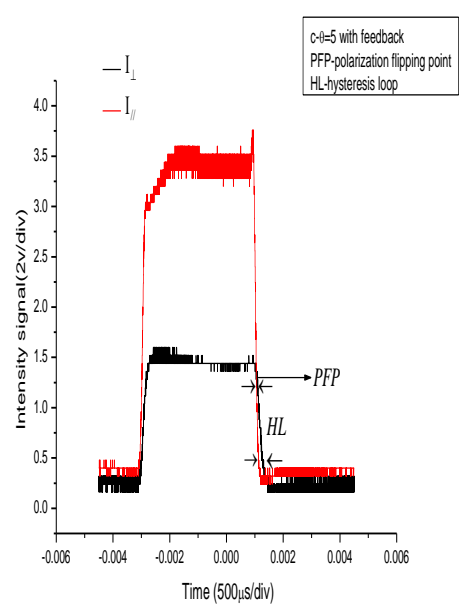

Figure 8-The intensities of $I_{/ /}$and $I_{\perp}$ with optical feedback at $\theta=5^{\circ}$

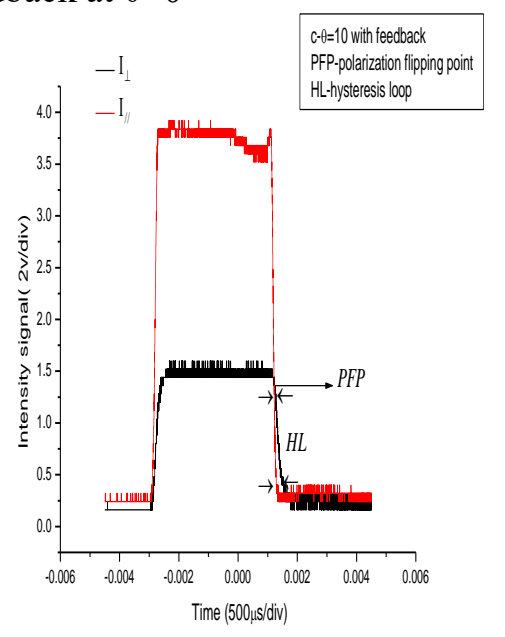

Figure 9-The intensities of $I_{/ /}$and $\mathrm{I}_{\perp}$ with optical feedback at $\theta=10^{\circ}$ 


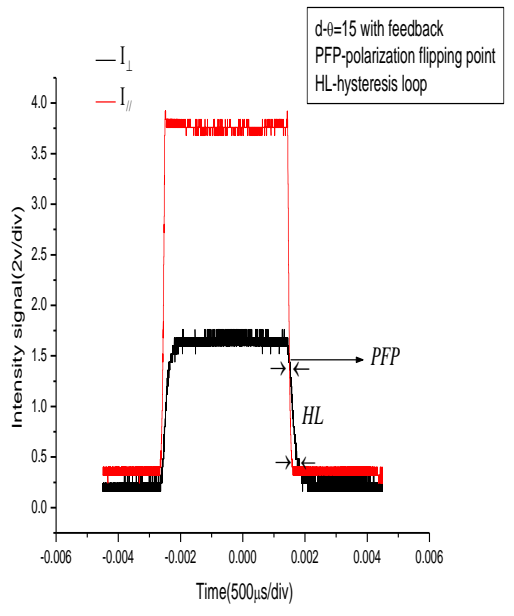

Figure 10 -The intensities of $\mathrm{I}_{/ /}$and $\mathrm{I}_{\perp}$ with feedback at $\theta=15^{\circ}$

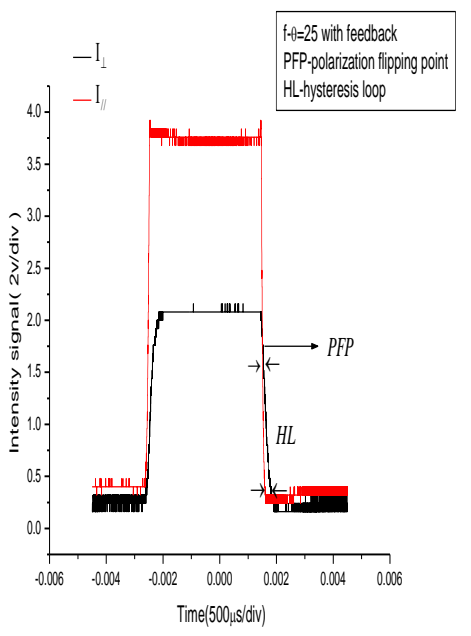

Figure 12-The intensities of $I_{/ /}$and $I_{\perp}$ with optical feedback at $\theta=25^{\circ}$

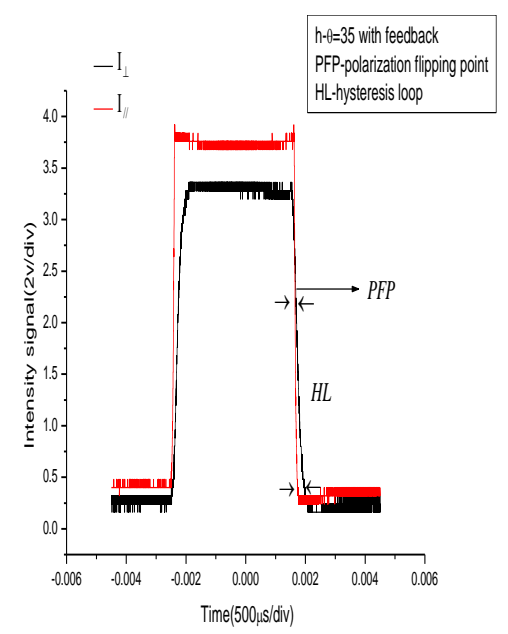

Figure 14 -The intensities of $I_{/ /}$and $I_{\perp}$ with optical feedback at $\theta=35^{\circ}$

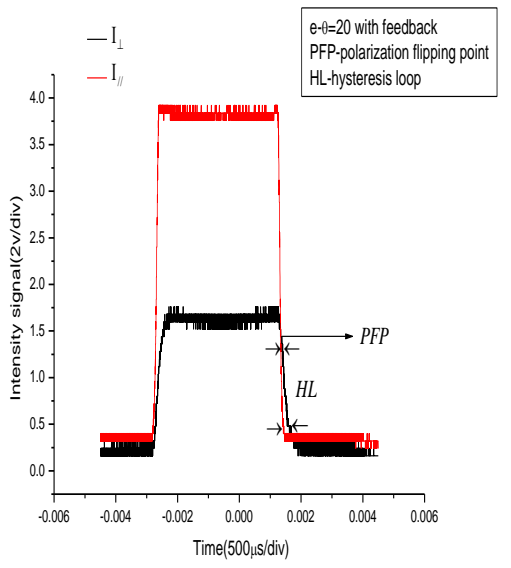

Figure 11-The intensities of $\mathrm{I}_{/ /}$optical and $\mathrm{I}_{\perp}$ with optical feedback at $\theta=20^{\circ}$

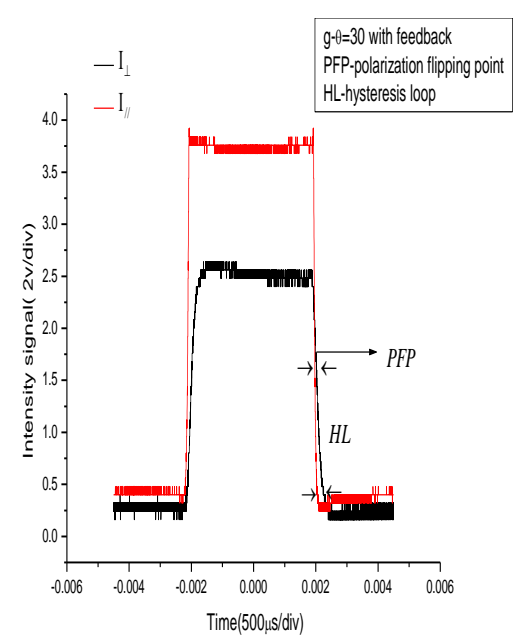

Figure 13-The intensities of $\mathrm{I}_{/ /}$ and $\mathrm{I}_{\perp}$ with optical feedback at $\theta=30^{\circ}$

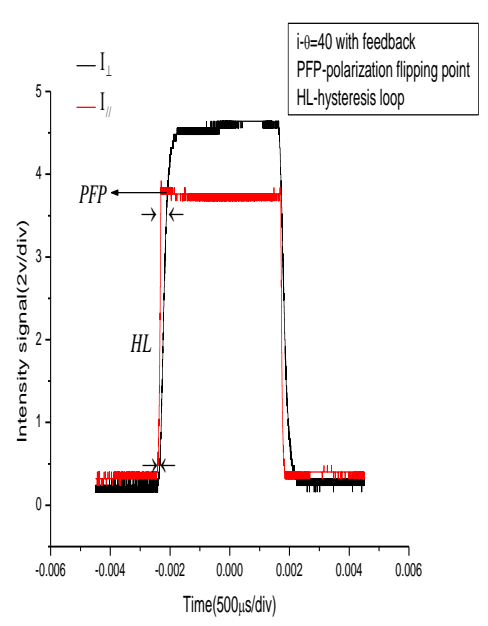

Figure 15- The intensities of $\mathrm{I}_{/ /}$ and $I_{\perp}$ with optical feedback at $\theta=40^{\circ}$ 


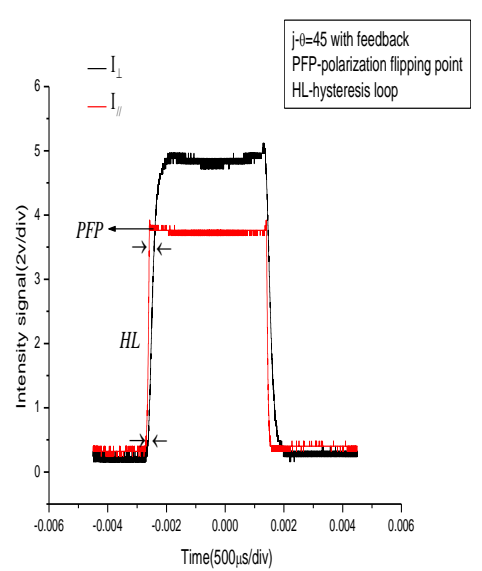

Figure 16 -The intensities of $\mathrm{I}_{/ /}$and $\mathrm{I}_{\perp}$ with optical feedback at $\theta=45^{\circ}$

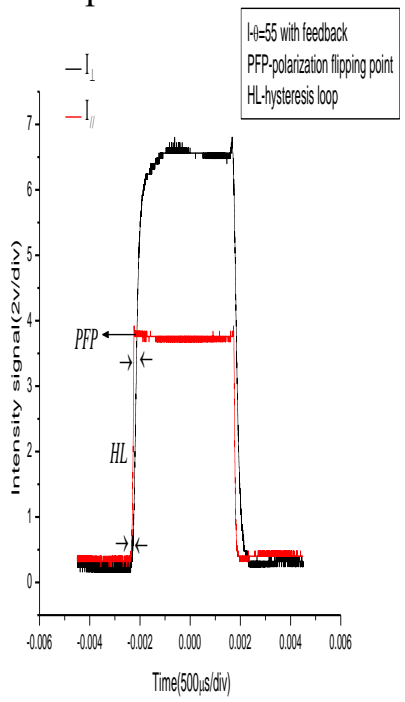

Figure 18-The intensities of $\mathrm{I}_{/ /}$and $\mathrm{I}_{\perp}$ with optical feedback at $\theta=55^{\circ}$

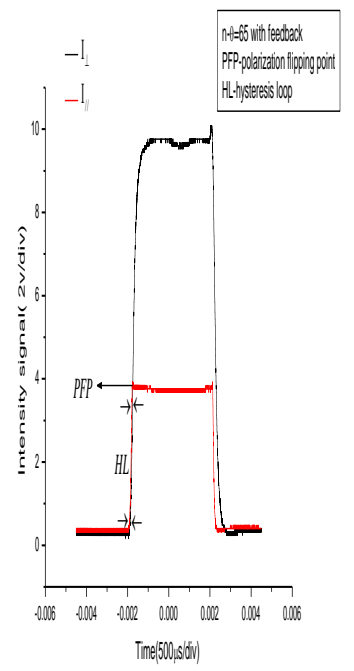

Figure 20-The intensities of $\mathrm{I}_{/ /}$and $\mathrm{I}_{\perp}$ with optical Figure 21 -The intensities of $\mathrm{I}_{/ /}$and $\mathrm{I}_{\perp}$ with optical feedback at $\theta=65^{\circ}$

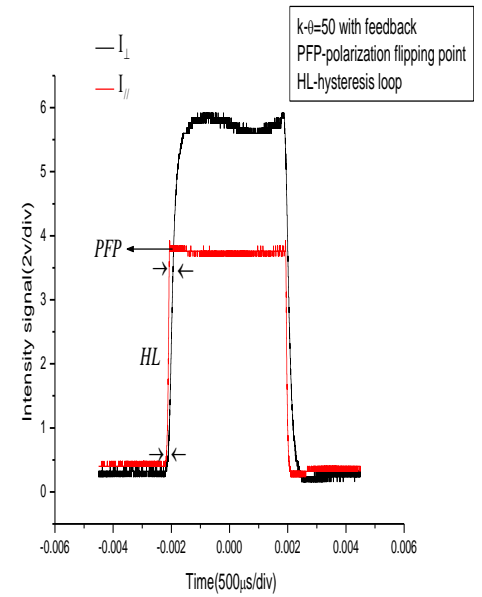
and $\mathrm{I}_{\perp}$ with optical feedback at $\theta=50^{\circ}$

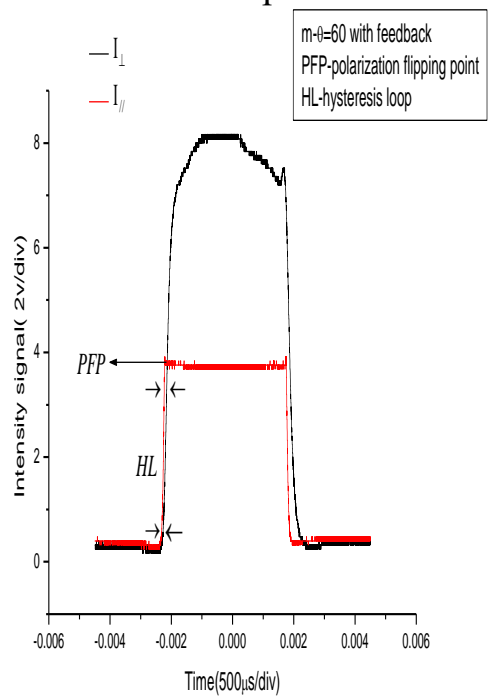

Figure 19- The intensities of $\mathrm{I}_{/ /}$and $\mathrm{I}_{\perp}$ with optical feedback at $\theta=60^{\circ}$

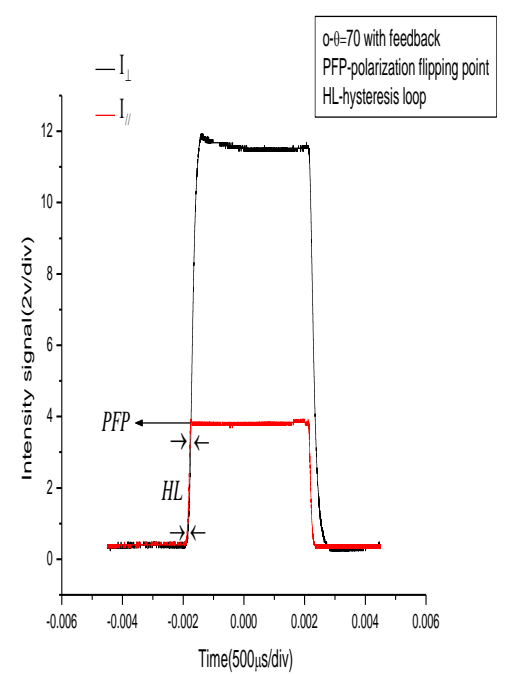

Figure 17- The intensities of $\mathrm{I}_{/ /}$ 


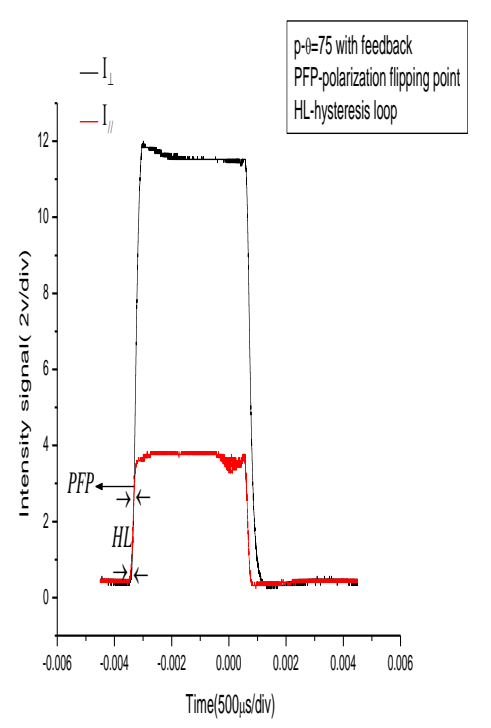

Figure 22- The intensities of $\mathrm{I}_{/ /}$and $\mathrm{I}_{\perp}$ with optical feedback at $\theta=75^{\circ}$

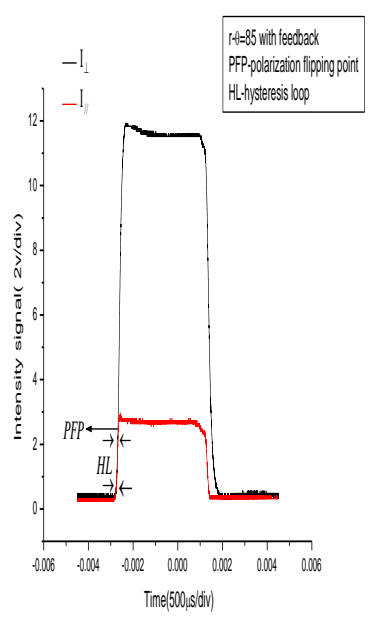

Figure 24-The intensities of $\mathrm{I}_{/ /}$and $\mathrm{I}_{\perp}$ with feedback at $\theta=85^{\circ}$

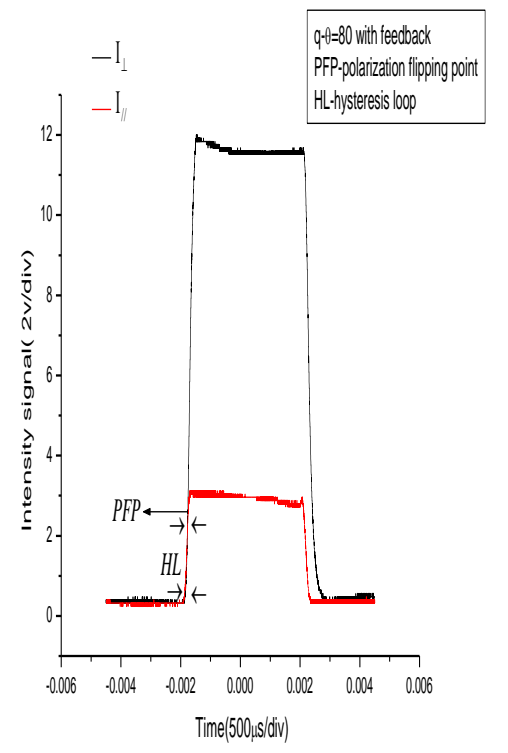

Figure 23- The intensities of $I_{/ /}$and $I_{\perp}$ with optical feedback at $\theta=80^{\circ}$

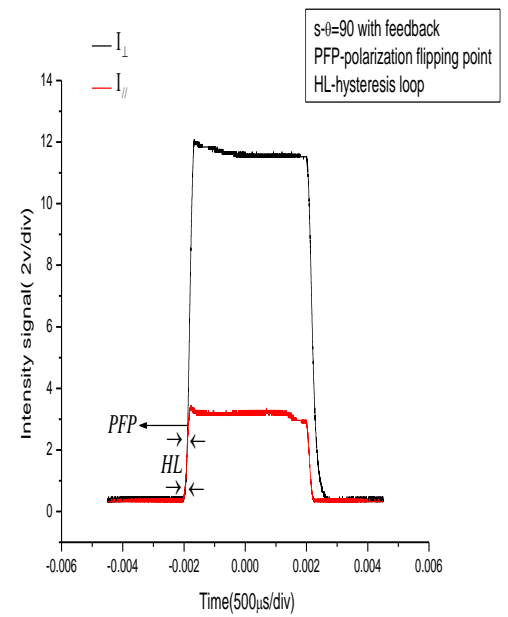

Figure 25 -The intensities of $\mathrm{I}_{/ /}$and $\mathrm{I}_{\perp}$ with optical optical feedback at $\theta=90^{\circ}$

\section{Conclusions}

We have proved that the polarization flipping point moves once to the right and another to the left by optical feedback. The intensity of the two components $\left(I_{/ /}\right.$and $\left.I_{\perp}\right)$ can be adjusted by polarizer rotation in the external laser cavity. The control on the vertical and horizontal polarizations is achieved by the polarizer in the external laser cavity. This is also a simple design that does not need a change in the structure of the laser or the adoption of complex optical elements. The control on the hysteresis loop between the vertical and horizontal polarizations can be performed by an optical feedback technique from the external cavity. 


\section{References}

1. Floch, A. L., Ropars, J. M. and Lenornamd, G. 1984. "Dynamics of laser eigenstates." Phys. Rev. Lett. 52: 918-921 (1984).

2. Panajotov K., Arizaleta M., Camarena M. and Thienpont H. 2004. "Polarization switching induced by short external cavity vertical-cavity surface-emitting lasers" Appl. Phys. Lett. 84: 2763-2765.

3. Chen, Y. C. and Liu, J. M. 1984. "Temperature-dependent polarization behavior of semiconductor lasers" Appl. Phys. Lett. 45: 731-733.

4. Seo, D. S., Park J. D., Mcinerney J. G. and Osinskia M. 1989. "Compound cavity modes in semiconductor lasers with asymmetric optical feedback" Appl. Phys. Lett. 54: 990-992.

5. Ropars, G., Floch, A. L. and Naour, R. L. 1992. "Polarization control mechanisms in vectorial bistable lasers for one-frequency systems" Phys. Rev. A. 46: 623-640.

6. Stephan, G. and Hugon, D. 1985. "Light polarization of a quasi-isotropic laser with optical feedback" Phys. Rev. Lett. 55: 703-706.

7. Yun, W., Yi-Dong, T., Shu-Lian, Z. and Yan, L. 2013. "Influence of Feedback Level on Laser Polarization in Polarized Optical Feedback" Chin. Phys. Lett. 30: 084201-1-4.

8. Ropars, G., Le Floch, A. and Le Naour, R. 1992. "Polarization control mechanisms in vectorial bistable lasers for one-frequency systems" Physical Review A, 46: 623-640.

9. Yun Wu, Shulian Zhang and Yan Li. 2013. "The intra-cavity phase anisotropy and the polarization flipping in HeNe laser" Optics Express, 21: 13684- 13690.

10. Acket, G.A., Lenstra, D., Boef, A.D. and Verbeek, B.H. 1984. "The influence of feedback intensity on longitudinal mode properties and optical noise in index-guided semiconductor lasers," Ieee J. Qe. 20: 1163-1169.

11. Tkach, R.W. and Chraplyvy, A.R. 1986. "Regimes of feedback effects in 1.5- $\mu \mathrm{m}$ distributed feedback lasers," J. Lightwave Technol. 4: 1655-1661.

12. Wang, W.M., Grattan, K.T.V., Palmer, A.W. and Boyle, W.J.O. 1994. "Self-mixing interference inside a single mode diode laser for optical sensing applications," J. Light wave Technol. 12: 1577-1587.

13. Wei, M., Shu-Lian, Z., Lian-Qing, Z., Jun, Z. and Yan, L. 2006. "Optical feedback characteristics is He-Ne dual frequency lasers" Chin. Phys. Lett. 32: 1188-1191. 